\title{
Electronic properties and magnetism of iron at the Earth's inner core conditions
}

L V. Pourovskii, T Miyake, Sergey Simak, A V. Ruban, L Dubrovinsky and Igor Abrikosov

\section{Linköping University Post Print}

\section{Tweet}

N.B.: When citing this work, cite the original article.

Original Publication:

L V. Pourovskii, T Miyake, Sergey Simak, A V. Ruban, L Dubrovinsky and Igor Abrikosov, Electronic properties and magnetism of iron at the Earth's inner core conditions, 2013, Physical Review B. Condensed Matter and Materials Physics, (87), 11.

http://dx.doi.org/10.1103/PhysRevB.87.115130

Copyright: American Physical Society http://www.aps.org/

Postprint available at: Linköping University Electronic Press http://urn.kb.se/resolve?urn=urn:nbn:se:liu:diva-91012 


\title{
Electronic properties and magnetism of iron at the Earth's inner core conditions
}

\author{
L. V. Pourovskii ${ }^{1,2}$ T. Miyake, ${ }^{3}$ S. I. Simak,${ }^{4}$ A. V. Ruban, ${ }^{5}$ L. Dubrovinsky, ${ }^{6}$ and I. A. Abrikosov ${ }^{4}$ \\ ${ }^{1}$ Centre de Physique Théorique, CNRS, École Polytechnique, 91128 Palaiseau, France \\ ${ }^{2}$ Swedish e-science Research Centre, Department of Physics, Chemistry and Biology, Linköping University, Linköping, Sweden \\ ${ }^{3}$ Nanosystem Research Institute, AIST, Tsukuba 305-8568, Japan \\ ${ }^{4}$ Department of Physics, Chemistry and Biology, Linköping University, Linköping, Sweden \\ ${ }^{5}$ Department of Materials Science and Engineering, Royal Institute of Technology, SE-10044 Stockholm, Sweden \\ ${ }^{6}$ Bayerisches Geoinstitut, Universität Bayreuth, 95440 Bayreuth, Germany
}

(Received 17 April 2012; published 21 March 2013)

\begin{abstract}
We employ state-of-the-art $a b$ initio simulations within the dynamical mean-field theory to study three likely phases of iron (hcp, fcc, and bcc) at the Earth's core conditions. We demonstrate that the correction to the electronic free energy due to correlations can be significant for the relative stability of the phases. The strongest effect is observed in bcc Fe, which shows a non-Fermi-liquid behavior, and where a Curie-Weiss behavior of the uniform susceptibility hints at a local magnetic moment still existing at $5800 \mathrm{~K}$ and $300 \mathrm{GPa}$. We predict that all three structures have sufficiently high magnetic susceptibility to stabilize the geodynamo.
\end{abstract}

DOI: $10.1103 /$ PhysRevB.87.115130

PACS number(s): 71.20.Be, 71.27.+a, 75.50.Bb, 91.60.Gf

\section{INTRODUCTION}

Being the main component of the Earth's core, iron attracts significant attention from a broad research community. Understanding its properties at ultrahigh pressure and temperature, ranging from studies of the core structure to modeling the geodynamo, is a long-term goal for condensedmatter physics and is essential for explaining geochemical observations, seismic data, and the theory of geomagnetism, to mention a few examples. In spite of all previous theoretical and experimental efforts, the crystal structure and properties of solid $\mathrm{Fe}$ at the Earth's inner core conditions remain a subject of intense debate. All three phases stable at low pressure-temperature conditions, namely, hexagonal closepacked (hcp), face-centered cubic (fcc), and body-centered cubic (bcc), have been suggested as possible crystal structures of iron or its alloys in the Earth's inner core. ${ }^{1-5}$

Theoretical simulations of iron at high pressures and temperatures generally rely on the picture of a wideband metal with insignificant local correlations. ${ }^{3-6}$ Indeed, under compression the overlap between localized states increases and so does the bandwidth $W$, while the local Coulomb repulsion $U$ between those states is screened more efficiently. The reduction of the $U / W$ ratio is used to rationalize the absence of electronic correlations beyond the standard localdensity approximation (LDA) at high-pressure conditions. The increase of the $3 d$-band width also results in the corresponding drop of the density of states at the Fermi energy, leading to the disappearance of the driving force for magnetism according to the Stoner criterion. In addition, even at the ambient pressure but very high temperature $T \gg T_{c}\left(T_{c}\right.$ is the Curie temperature, which is $1043 \mathrm{~K}$ in $\alpha$-Fe), local magnetic moments are expected to be suppressed due to one-electron Stoner-type excitations. Thus, when extremely high pressure and temperature are simultaneously applied, disappearance of the local magnetic moment seems to be inevitable. Due to these considerations iron at the Earth's inner core conditions has been modeled as nonmagnetic within LDA-based approaches. The results of recent works of Sola et al., ${ }^{7,8}$ who applied fixed-nodeapproximation quantum Monte Carlo techniques to compute the equation of state and the melting temperature of hcp Fe at extreme conditions, are in good agreement with previous LDAbased simulations and thus strengthen the above argument, at least in the case of the hcp phase. On the other hand, Glazyrin et al. ${ }^{9}$ have just demonstrated the importance of correlation effects in hcp iron revealed by an electronic topological transition induced at a pressure of $40 \mathrm{GPa}$ and room temperature.

So are electronic correlations important at extreme conditions, and can they lead to qualitatively new phenomena? To address these questions we have investigated the impact of correlations on the electronic structure, magnetic properties, and thermodynamic stability of iron by performing $a b$ initio simulations of the bcc, fcc, and hcp phases for a volume of $7.05 \AA$ /atom, corresponding to the pressures expected in the inner Earth's inner core, and temperatures up to $5800 \mathrm{~K}$ (The $c / a$ ratio in hcp Fe was fixed at 1.60). ${ }^{10}$ We employ a state-of-the-art fully self-consistent technique ${ }^{11,12}$ combining the full-potential linearized augmented plain-wave (FLAPW) band structure method ${ }^{13}$ with the dynamical mean-field theory (DMFT) ${ }^{14}$ treatment of the on-site Coulomb repulsion between Fe $3 d$ states. A combination of LDA and DMFT was applied earlier to study thermodynamic stability ${ }^{15}$ and to describe the magnetic properties ${ }^{16}$ of paramagnetic bcc Fe at ambient pressure.

\section{METHOD}

In our LDA + DMFT calculations Wannier-like functions for the Fe $3 d$ shell were constructed by projecting local orbitals onto a set of FLAPW Bloch states located within the energy window from -10.8 to $4 \mathrm{eV}$ relative to the Fermi level $E_{F}$ (details of the projection procedure can be found in Ref. 11).

We then introduced the calculated local Coulomb interaction in the density-density form acting between those Wannier orbitals. In order to evaluate the strength of the on-site electron repulsion on the $\mathrm{Fe} 3 d$ shell we employed the constrained random-phase-approximation (cRPA) method. ${ }^{17,18}$ The calculated Coulomb $(U)$ and exchange $(J)$ interaction matrices are well approximated by a spherically symmetric form used in the subsequent calculations, with the parameter $U$ (the 
Slater parameter $F_{0}$ ) equal to $3.15,3.04$, and $3.37 \mathrm{eV}$ and the Hund's rule coupling $J$ equal to $0.9,0.9$, and $0.93 \mathrm{eV}$ for the bcc, fcc, and hcp phases, respectively. These results are in general agreement with previous calculations of $U$ in compressed Fe. ${ }^{19}$ We employed the around mean-field form ${ }^{20}$ of the double-counting correction term throughout.

The resulting many-body problem has been treated within the DMFT framework with the quantum impurity problem solved by the numerically exact continuous-time hybridization expansion quantum Monte Carlo (CT-QMC) method $^{21}$ using $5 \times 10^{8}$ CT-QMC moves with a measurement performed after each 200 moves. After completing the DMFT cycle we calculated the resulting density matrix in the Bloch states' basis, which was then used to recalculate the charge density in the next iteration, as described in Ref. 12. To obtain the spectral function at the real axis we employed a stochastic version of the maximum entropy method ${ }^{22}$ for analytical continuation. In order to compute the magnetic susceptibility in uniform fields we performed LDA + DMFT simulations with the Kohn-Sham eigenstates split by the magnetic field $H=0.005 \mathrm{eV} / \mu_{B}$ directed along the $z$ axis and then computed $\chi=M / H$ from the resulting small magnetic moment $M \sim 0.01 \mu_{B}$.

\section{RESULTS AND DISCUSSION}

In Fig. 1 we display the LDA + DMFT k-resolved spectral functions $A(\mathbf{k}, E)$ for the three phases obtained for a temperature of $5800 \mathrm{~K}$. First, one may notice that in hcp $\mathrm{Fe}$ the electronic states in the vicinity of $E_{F}$ are sharp [their
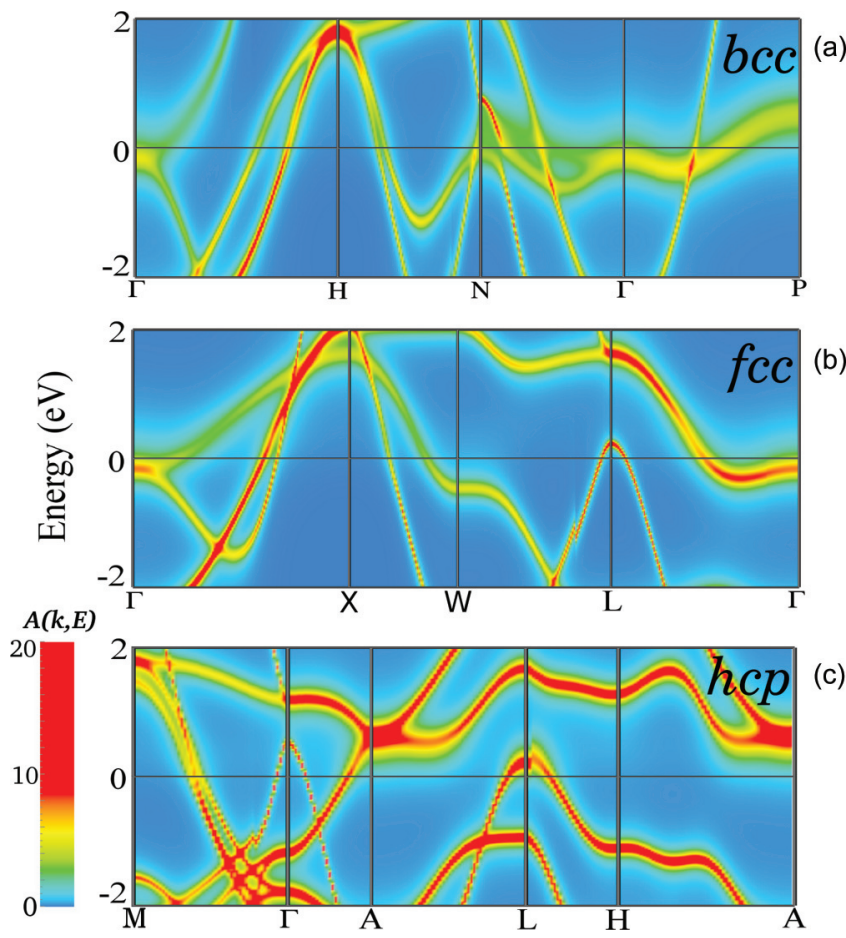

FIG. 1. (Color online) The LDA + DMFT k-resolved spectral function $A(\mathbf{k}, E)\left(V_{a t} / \mathrm{eV}\right)$ for (a) bcc, (b) fcc, and (c) hcp Fe at volume $V_{a t}=7.05 \AA^{3} /$ atom and temperature $5800 \mathrm{~K}$. A nonquasiparticle $e_{g}$ band is seen in the vicinity of the Fermi energy along the $N-\Gamma-P$ path in (a).

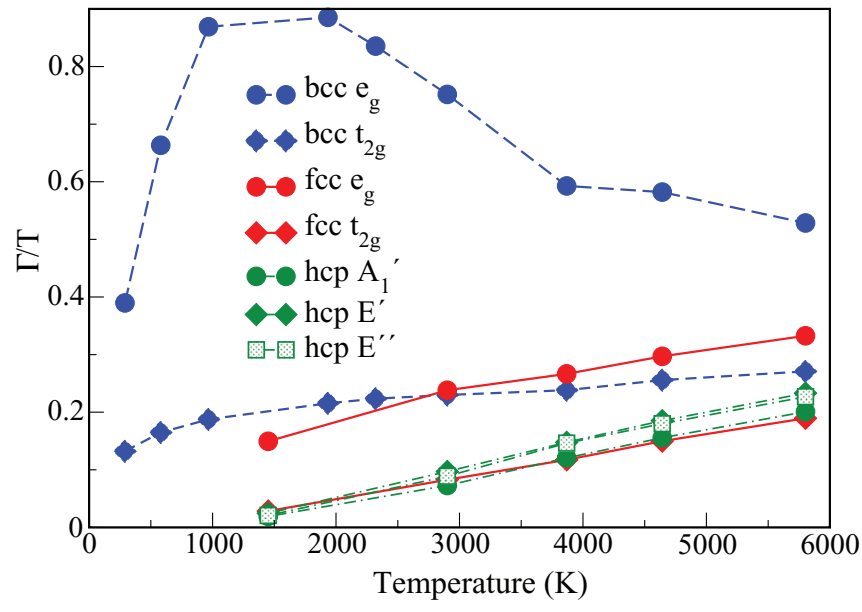

FIG. 2. (Color online) The ratio of the inverse quasiparticle lifetime $\Gamma$ to temperature $T$ vs. $T$. The solid red, dashed blue, and dash-dotted green curves correspond to $3 d$ states in fcc, bcc, and hcp $\mathrm{Fe}$, respectively. They are split by the crystal field into $t_{2 g}$ (diamonds) and $e_{g}$ (circles) representations in the cubic (bcc and fcc) phases and two doubly degenerate representations ( $E^{\prime}$ and $E^{\prime \prime}$, shown by diamonds and squares, respectively) and one singlet representation ( $A_{1}^{\prime}$, circles) in the hcp phase, respectively. A nonlinear behavior of $\Gamma / T$ for bcc Fe $e_{g}$ states is clearly seen.

red color indicates a high value of $A(\mathbf{k}, E)]$, hence $\epsilon$-Fe exhibits the typical behavior of a Fermi liquid (FL) with large quasiparticle lifetimes in the vicinity of $E_{F}$. In contrast, the bcc phase features a low-energy $e_{g}$ band along the $N-\Gamma-P$ path that is strongly broadened, thus indicating destruction of quasiparticle states. fcc $\mathrm{Fe}$ is in an intermediate state, with some broadening noticeable in the $e_{g}$ bands at $E_{F}$ in the vicinity of the $\Gamma$ and $W$ points.

To quantify the degree of non-Fermi-liquid (non-FL) behavior we have evaluated the inverse quasiparticle lifetime $\Gamma=-Z \operatorname{Im}\left[\Sigma\left(i 0^{+}\right)\right]$, where the quasiparticle residue $Z^{-1}=$ $1-\left.\frac{\partial \operatorname{Im} \Sigma(i \omega)}{\partial \omega}\right|_{\omega \rightarrow 0^{+}}$by extrapolating the imaginary-frequency self-energy $\Sigma(i \omega)$ to zero. In the FL regime $\Gamma$ scales as $T^{2}$; hence $\Gamma / T$ vs $T$ is linear. In Fig. 2 we display the temperature evolution of $\Gamma / T$ for the relevant irreducible representations of the Fe $3 d$ shell in all three phases. One may see that $\Gamma / T$ in hcp Fe exhibits a linear increase typical for a FL up to temperatures expected in the Earth's inner core. In contrast, $\Gamma / T$ for the bcc iron $e_{g}$ states features a linear and steep rise for $T<1000 \mathrm{~K}$ and then behaves nonlinearly, indicating the noncoherent nature of those states at high temperatures. The bcc Fe $t_{2 g}$ and fcc $\mathrm{Fe} e_{g}$ electrons are in an intermediate situation, with some noticeable deviations from the FL behavior.

The tendency of bcc $e_{g}$ states to a non-FL behavior has been noted before for ambient conditions and has been explained by a smaller effective bandwidth of the "localized" $e_{g}$ band compared to the $t_{2 g}$ one. ${ }^{23}$ We have evaluated the one-electron kinetic energy of the $e_{g}$ and $t_{2 g}$ bands as $E_{b}=\int_{-\infty}^{E_{F}} D(E)(E-$ $C) d E$, where $D(E)$ is the corresponding LDA partial density of states (PDOS) and $C$ is the central weight of the band. Resulting $E_{b}$ for the $e_{g}$ and $t_{2 g}$ bands in the bcc (fcc) phases are equal to $-1.05(-1.01)$ and $-1.08(-1.20) \mathrm{eV}$, respectively. One may see that the difference in kinetic energy between the 

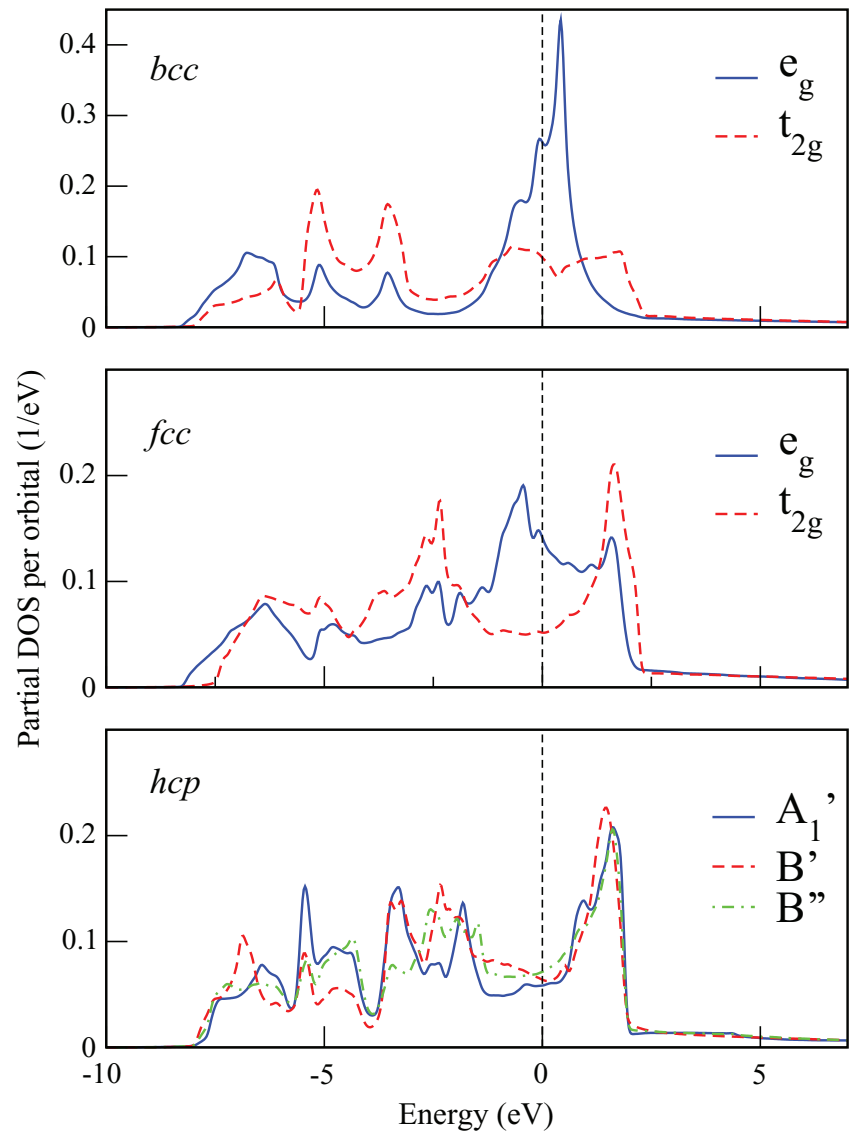

FIG. 3. (Color online) Partial LDA densities of states (PDOS) for the irreducible representations of $\mathrm{Fe} 3 d$ states for the three phases with a volume of $7.05 \AA^{3}$ /atom. The large peak in the vicinity of $E_{F}$ in bcc Fe $e_{g}$ PDOS is due to a van Hove singularity.

$e_{g}$ and $t_{2 g}$ bands in bcc Fe is rather small and in fact even smaller than the corresponding difference in the fcc phase. Hence it can hardly explain the observed qualitatively distinct non-FL behavior of the $e_{g}$ states in bcc. It has been pointed out $^{24,25}$ that a van Hove singularity in one of the bcc Fe $e_{g}$ bands leads to the formation of a narrow peak in the corresponding PDOS in the vicinity of $E_{F}$ (Fig. 3). A large peak in PDOS located at $E_{F}$ leads to suppression of the low-energy hopping and to the corresponding enhancement of correlations, as has been recently pointed out for the case of $\mathrm{Sr}_{2} \mathrm{RuO}_{4} \cdot{ }^{26}$ A similar suppression is observed in the $e_{g}$ hybridization function in bcc $\mathrm{Fe}$, as one may see in Fig. 4.

Having demonstrated the impact of correlation effects on the Fe electronic structure, we now focus on its consequences for the $\mathrm{Fe}$ phase stability and magnetism. To evaluate the impact of correlation effects on the relative stability of the three phases we have computed the corresponding correction to the fixed-lattice free energy by employing a coupling-constant integration approach (see, e.g., Ref. 27). We define the free energy as $F_{\lambda}=-\frac{1}{\beta} \ln \operatorname{Tr}\left\{\exp \left[-\beta\left(H_{0}+\lambda H_{1}\right)\right]\right\}$, corresponding to a given value of the coupling $\lambda \in[0: 1]$, where $H_{0}$ is the one-electron (LDA) part of the Hamiltonian and $H_{1}$ is the interacting part equal to the difference between the Hubbard term $H_{U}$ and the double-counting correction $E_{d c}$. The coupling constant integration leads to the following expression for the

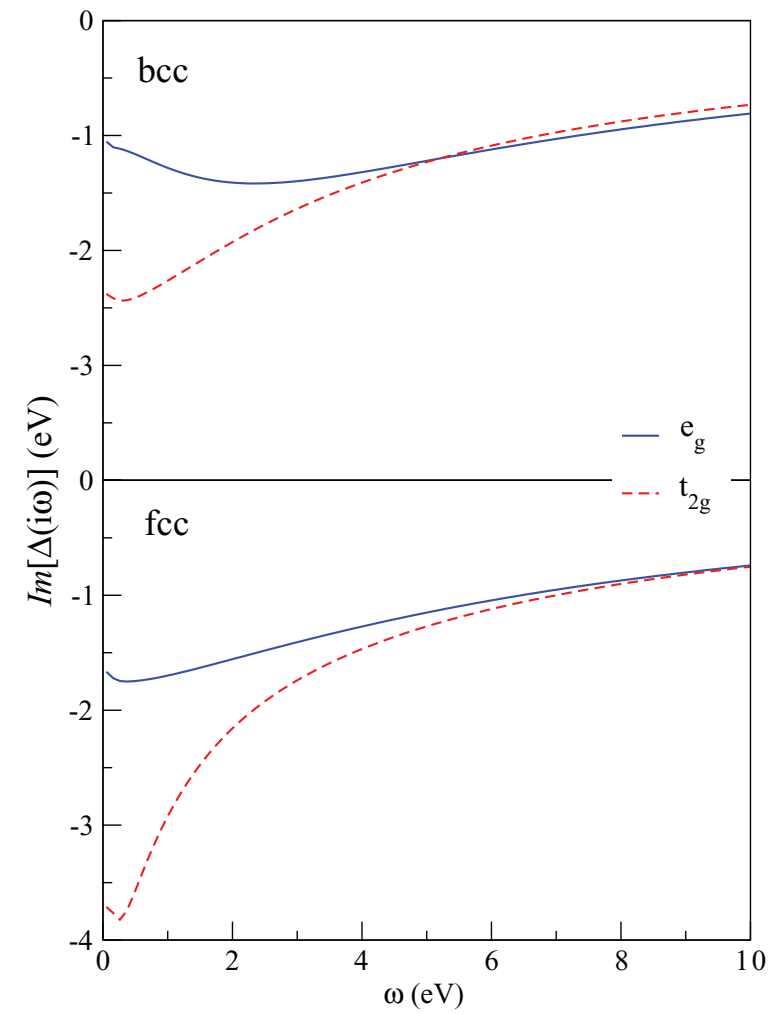

FIG. 4. (Color online) Imaginary part of the hybridization function $\Delta$ as a function of imaginary frequency for the $e_{g}$ and $t_{2 g}$ states at the first DMFT iteration. One may clearly see a decrease in $|\operatorname{Im} \Delta(i \omega)|$ at $\omega<2 \mathrm{eV}$ of the $e_{g}$ states in bcc Fe. However, at higher energies $(\omega>5 \mathrm{eV})$ the $e_{g}$ hybridization function decays slowly and becomes larger than the $t_{2 g}$ one. The overall one-electron kinetic energies of the $e_{g}$ and $t_{2 g}$ states in bcc Fe have similar values, as explained in the main text. In contrast, in the fcc phase $|\Delta|$ grows monotonously with decreasing $\omega$ for both $e_{g}$ and $t_{2 g}$

many-body correction:

$$
\Delta F=F-F_{0}=\int_{0}^{1} \frac{\left\langle\lambda H_{1}\right\rangle}{\lambda} d \lambda .
$$

In derivation of Eq. (1) we neglected the $\lambda$ dependence of the one-electron part and hence the charge-density renormalization due to many-body effects. However, we verified that the correction to the total energy due to the charge-density self-consistency is rather small and within our error bars.

To obtain $\Delta F$ we have computed $\frac{\left\langle\lambda H_{1}\right\rangle_{\lambda}}{\lambda}$ for a discrete set of values of $\lambda$ ranging from 0 to 1 by performing LDA + DMFT simulations with the Coulomb interaction scaled accordingly and evaluating $\left\langle\lambda H_{U}\right\rangle_{\lambda}$ in accordance with the Migdal formula. (We calculated $\left.\frac{\left\langle\lambda H_{1}\right\rangle_{\lambda}}{\lambda}\right|_{\lambda=0}$ analytically, as in this case it is equal to the Hartree-Fock approximation to $\left\langle H_{U}\right\rangle$ computed with the LDA density matrix minus the double-counting correction. The resulting value of $\left.\frac{\left\langle\lambda H_{1}\right\rangle_{\lambda}}{\lambda}\right|_{\lambda=0}$ is small, of the order of 0.1 mRy.) Then we integrated $\frac{\left\langle\lambda H_{1}\right\rangle_{\lambda}}{\lambda}$ over $\lambda$ numerically in order to obtain $\Delta F$ and its error bars.

The resulting many-body correction to the electronic free energy is displayed in Fig. 5 for temperatures of 2900 and $5800 \mathrm{~K}$. There we also show the corresponding correction to the total energy $\Delta E=E_{\mathrm{DMFT}}-E_{\mathrm{LDA}}$, where $E_{\mathrm{DMFT}}$ was 


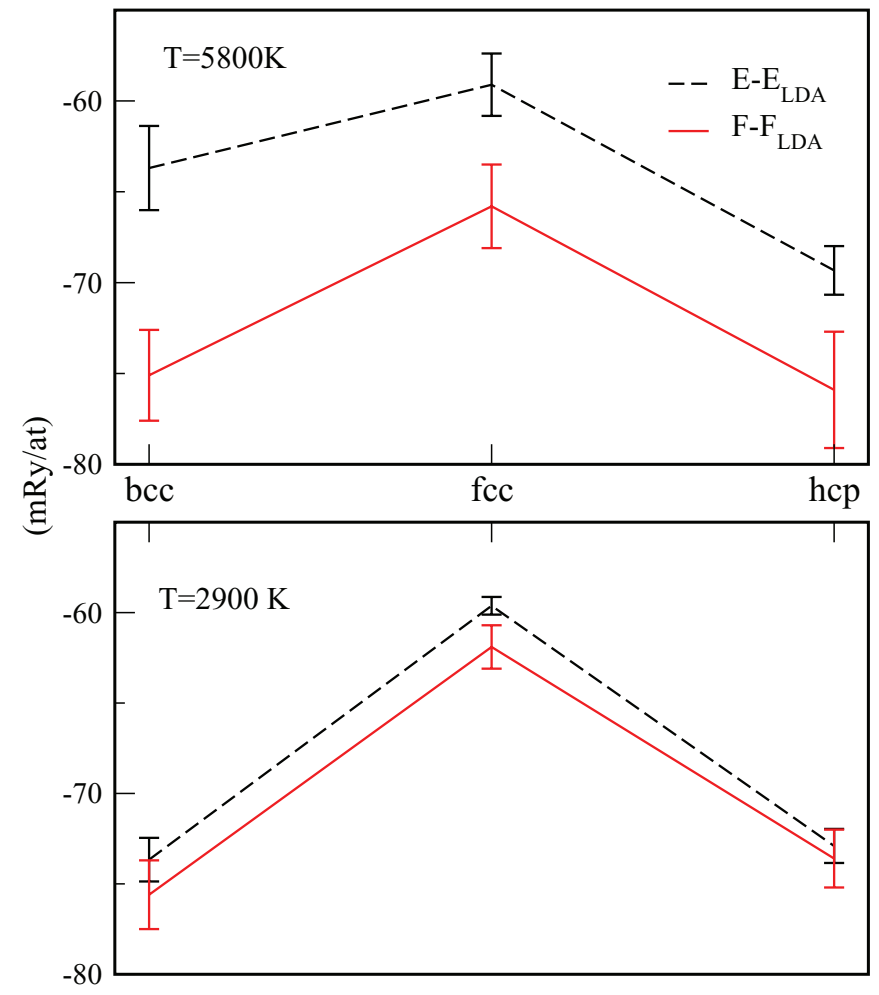

FIG. 5. (Color online) Many-body correction to the total (black dashed line) and free (red solid line) energy for the three phases of Fe with a volume of $7.05 \AA$ /atom at (top) $T=5800 \mathrm{~K}$ and (bottom) $2900 \mathrm{~K}$. The error bars are due to the CT-QMC stochastic error.

computed in accordance with Eq. (3) of Ref. 12. Within our error bars the magnitude of $\Delta F$ is the same for bcc and hcp Fe, which are suggested as stable phases of iron ${ }^{4}$ and iron-based alloys ${ }^{1,2}$ at the Earth's inner core conditions. The magnitude of $\Delta F$ is at least several millirydbergs smaller in the case of fcc Fe, showing that the many-body correction may significantly affect relative energy differences among iron phases at the Earth's core conditions. One may also notice that the entropic contribution $T \Delta S=\Delta E-\Delta F$ becomes much more significant at the higher temperature, and its contribution is almost twice as large in the case of the bcc phase compared with the other two.

The application of the LDA + DMFT theory has the most important consequences for the understanding of magnetic properties of Fe at the Earth's core conditions. In Fig. 6 we display the temperature evolution of the uniform magnetic susceptibility $\chi$ in the range of temperatures from 1100 to $5800 \mathrm{~K}$. One may notice that in the fcc and hep phases the susceptibility exhibits a temperature-independent Pauli behavior expected for a FL (a small decrease in $\chi$ at lower temperatures observed in hcp $\mathrm{Fe}$ is due to a dip in its one-electron DOS in the vicinity of the Fermi level; see Fig. 3). In contrast, $\chi$ of bcc Fe features a clear inverse-temperature dependence and can be very well described by the Curie-Weiss (CW) law $\chi=\frac{1}{3} \frac{\mu_{\text {eff }}^{2}}{T+\Theta}$, with $\mu_{\text {eff }}=2.6 \mu_{B}$ and $\Theta=1396 K$ (see inset in Fig. 6).

One may relate the apparent $\mathrm{CW}$ behavior of the magnetic susceptibility in bcc Fe to the high peak at $E_{F}$ present in its LDA DOS (Fig. 3), which can lead to a strongly temperature-

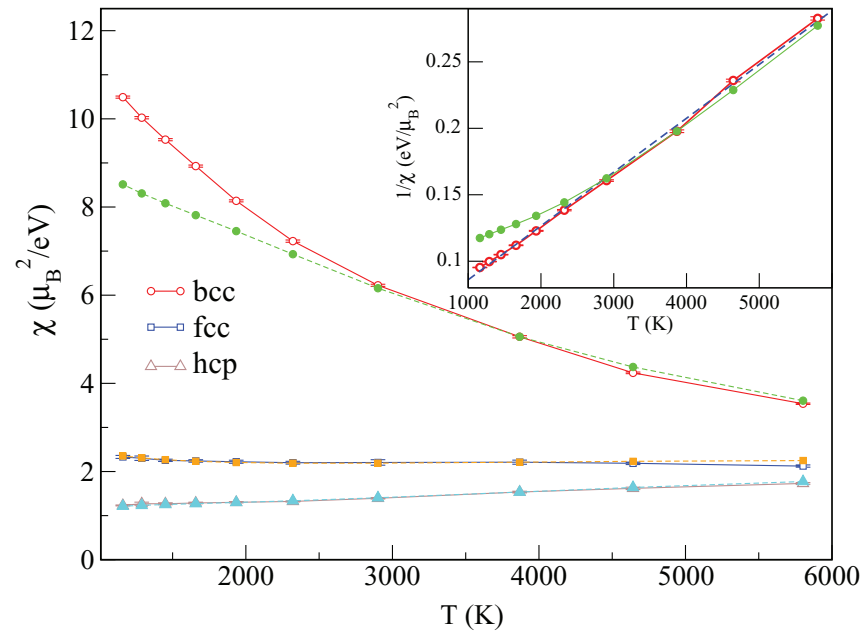

FIG. 6. (Color online) The uniform magnetic susceptibility in a paramagnetic state vs temperature. The error bars are due to the CT-QMC stochastic error. The dashed lines with corresponding solid symbols are fits to the enhanced Pauli law; see the text. In the inset the inverse uniform magnetic susceptibility of bcc Fe is shown in red (open circles), and the blue dashed and green solid (solid circles) lines are fits to the Curie-Weiss and enhanced Pauli laws, respectively.

dependent Pauli (band) susceptibility. We have computed the Stoner-enhanced Pauli susceptibilities $\chi_{s t}=\chi_{0} /\left(1-I \chi_{0}\right)$ for all three phases, where $I$ is the Stoner parameter and $\chi_{0}$ is the bare uniform Pauli susceptibility computed by LDA FLAPW calculations for finite temperatures with a small external magnetic field switched on. We fitted $I$ to reproduce the corresponding values of LDA + DMFT $\chi$ at $T=3800 \mathrm{~K}$; the resulting values of $I$ are $0.44,0.53$, and $0.54 \mathrm{eV}$ in the bcc, fcc, and hcp phases, respectively. Obtained $\chi_{s t}$ reproduce very well the LDA + DMFT magnetic susceptibilities of fcc and hcp Fe, thus confirming the FL nature of these phases. In bcc Fe $\chi_{s t}$ describes well the CW-like behavior of LDA + DMFT susceptibility in the range from 3000 to $5800 \mathrm{~K}$. However, $\chi_{s t}$ deviates from the LDA + DMFT susceptibility significantly at lower temperatures $T$, which are small compared to the characteristic width of the peak at $E_{F}$ in the LDA DOS; see Fig. 6.

An alternative and more interesting source for the apparent $\mathrm{CW}$ behavior of the uniform susceptibility in bcc Fe can be a local magnetic moment surviving in this phase up to Earth's core temperatures. The existence of a local magnetic moment of the constant magnitude also provides a natural explanation for the inverse bcc Fe susceptibility exhibiting the same linear temperature dependence in the whole range from 1100 to $5800 \mathrm{~K}$, with no significant deviations or noticeable peculiarities (see inset in Fig 6).

The value of calculated uniform susceptibility in SI units at temperature $T=5800 \mathrm{~K}$ is equal to $1.7 \times 10^{-4}, 2.0 \times 10^{-4}$, and $3.5 \times 10^{-4}$ for hcp, fcc, and bcc Fe, respectively. We would like to underline that our calculated uniform magnetic susceptibilities in all three phases of Fe are sufficiently high to be important for models of the Earth's core dynamics and geodynamo (see, e.g., Ref. 28). An inner core with a paramagnetic susceptibility in the range $10^{-3}-10^{-4}$ SI units and with a paramagnetic relaxation time acting slower than 
field changes coming from the outer core could also attenuate short-frequency fluctuations and become an important factor stabilizing the geodynamo, ${ }^{29}$ just as an electrically conducting inner core could stabilize the geodynamo because the inner core would have a magnetic diffusion constant independent of the outer core. ${ }^{30,31}$

In conclusion, we have carried out a theoretical investigation of the role of electronic correlations in the bcc, fcc, and hcp phases of Fe at the Earth's inner core conditions using a fully self-consistent LDA + DMFT approach. We have found that the fcc and hep phases remain in a Fermi-liquid state, while bcc Fe features a non-Fermi-liquid behavior. We have evaluated a correction to the electronic free energy due to many-body effects and found that it affects significantly the relative free-energy differences, penalizing the fcc phase. Most interestingly, our results suggest that a local magnetic moment may exist in the bcc phase at the inner core conditions and that magnetic susceptibilities in all three phases of $\mathrm{Fe}$ are sufficiently high to stabilize the geodynamo. Thus, new models of the geodynamo as well as the core structure and elasticity should include the magnetism of the Earth's core, the effect of which has not yet been considered.

\section{ACKNOWLEDGMENTS}

We are grateful to J. Mravlje, V. Vildosola, and A. Georges for useful discussions. We acknowledge the funding provided by the Swedish e-science Research Centre (SeRC), the Swedish Research Centre for Advanced Functional Materials (AFM), the Linköping Linnaeus Initiative for Novel Functional Materials (LiLI-NFM), SRL Grant No. 10-0026 from the Swedish Foundation for Strategic Research (SSF), Swedish Research Council (VR) Grant No. 621-2011-4426, and PHD DALEN 2012 Project 26228RM, as well as financial support from the German Science Foundation (DFG) and the German Ministry for Education and Research (BMBF). Calculations have been performed using the facilities of the National Supercomputer Centre (NSC) in Linköping and the High Performance Computing Center North (HPC2N) at the Swedish National Infrastructure for Computing (SNIC).
${ }^{1}$ J.-F. Lin, D. L. Heinz, A. J. Campbell, J. M. Devine, and G. Shen, Science 295, 313 (2002).

${ }^{2}$ L. Dubrovinsky, N. Dubrovinskaia, O. Narygina, I. Kantor, A. Kuznetzov, V. B. Prakapenka, L. Vitos, B. Johansson, A. S. Mikhaylushkin, S. I. Simak et al., Science 316, 1880 (2007).

${ }^{3}$ A. B. Belonoshko, R. Ahuja, and B. Johansson, Nature (London) 424, 1032 (2003).

${ }^{4}$ L. Vočaldo, D. Alfè, M. J. Gillan, I. G. Wood, J. P. Brodholt, and G. D. Price, Nature (London) 424, 536 (2003).

${ }^{5}$ A. S. Mikhaylushkin, S. I. Simak, L. Dubrovinsky, N. Dubrovinskaia, B. Johansson, and I. A. Abrikosov, Phys. Rev. Lett. 99, 165505 (2007).

${ }^{6}$ L. Stixrude, Phys. Rev. Lett. 108, 055505 (2012).

${ }^{7}$ E. Sola, J. P. Brodholt, and D. Alfè, Phys. Rev. B 79, 024107 (2009).

${ }^{8}$ E. Sola and D. Alfè, Phys. Rev. Lett. 103, 078501 (2009).

${ }^{9}$ K. Glazyrin, L. V. Pourovskii, L. Dubrovinsky, O. Narygina, C. McCammon, B. Hewener, V. Schünemann, J. Wolny, K. Muffler, A. I. Chumakov et al., Phys. Rev. Lett. 110, 117206 (2013).

${ }^{10}$ S. Ono, T. Kikegawa, N. Hirao, and K. Mibe, Am. Mineral. 95, 880 (2010).

${ }^{11}$ M. Aichhorn, L. Pourovskii, V. Vildosola, M. Ferrero, O. Parcollet, T. Miyake, A. Georges, and S. Biermann, Phys. Rev. B 80, 085101 (2009).

${ }^{12}$ M. Aichhorn, L. Pourovskii, and A. Georges, Phys. Rev. B 84, 054529 (2011).

${ }^{13}$ P. Blaha, K. Schwarz, G. Madsen, D. Kvasnicka, and J. Luitz, WIEN2k, an Augmented Plane Wave + Local Orbitals Program for Calculating Crystal Properties (Technische Universitat Wien, Vienna, 2001).
${ }^{14}$ A. Georges, G. Kotliar, W. Krauth, and M. J. Rozenberg, Rev. Mod. Phys. 68, 13 (1996).

${ }^{15}$ I. Leonov, A. I. Poteryaev, V. I. Anisimov, and D. Vollhardt, Phys. Rev. Lett. 106, 106405 (2011).

${ }^{16}$ A. I. Lichtenstein, M. I. Katsnelson, and G. Kotliar, Phys. Rev. Lett. 87, 067205 (2001).

${ }^{17}$ F. Aryasetiawan, M. Imada, A. Georges, G. Kotliar, S. Biermann, and A. I. Lichtenstein, Phys. Rev. B 70, 195104 (2004).

${ }^{18}$ T. Miyake and F. Aryasetiawan, Phys. Rev. B 77, 085122 (2008).

${ }^{19}$ M. Cococcioni and S. de Gironcoli, Phys. Rev. B 71, 035105 (2005).

${ }^{20}$ M. T. Czyżyk and G. A. Sawatzky, Phys. Rev. B 49, 14211 (1994).

${ }^{21}$ E. Gull, A. J. Millis, A. I. Lichtenstein, A. N. Rubtsov, M. Troyer, and P. Werner, Rev. Mod. Phys. 83, 349 (2011).

${ }^{22}$ K. S. D. Beach, arXiv:cond-mat/0403055 (unpublished).

${ }^{23}$ A. A. Katanin, A. I. Poteryaev, A. V. Efremov, A. O. Shorikov, S. L. Skornyakov, M. A. Korotin, and V. I. Anisimov, Phys. Rev. B 81, 045117 (2010).

${ }^{24}$ R. Maglic, Phys. Rev. Lett. 31, 546 (1973).

${ }^{25}$ V. Y. Irkhin, M. I. Katsnelson, and A. V. Trefilov, J. Phys.: Condens. Matter 5, 8763 (1993).

${ }^{26}$ J. Mravlje, M. Aichhorn, T. Miyake, K. Haule, G. Kotliar, and A. Georges, Phys. Rev. Lett. 106, 096401 (2011).

${ }^{27}$ P. Nozières, Theory of Interacting Fermi Systems (Benjamin, New York, 1963).

${ }^{28}$ J. Aubert, S. Labrosse, and C. Poitou, Geophys. J. Int. 169, 1414 (2009).

${ }^{29}$ B. M. Clement and L. Stixrude, Earth Planet. Sci. Lett. 130, 75 (1995).

${ }^{30}$ S. Gilder and J. Glen, Science 279, 72 (1998).

${ }^{31}$ R. Hollerbach and C. Jones, Nature (London) 365, 541 (1993). 